\title{
Development of ReproKnow, a reproductive knowledge assessment for women with rheumatic diseases
}

\author{
Mehret Birru Talabi ${ }^{1 *} \mathbb{D}$, Megan E. B. Clowse ${ }^{2}$, Susan J. Blalock ${ }^{3}$, Galen Switzer ${ }^{4}$, Lan Yu ${ }^{4}$, Alaina Chodoff and \\ Sonya Borrero ${ }^{4,6}$
}

\begin{abstract}
Background: The objective of this study was to develop an assessment tool, ReproKnow, to evaluate the reproductive health knowledge of women with a wide range of rheumatic diseases.

Methods: The 10-item multiple-choice questionnaire was developed with feedback from a panel of content experts and female patients with rheumatic diseases. Construct validity using known-groups analysis was evaluated through comparison of median total Reproknow scores between rheumatology fellows and nurses. Female patients aged 18-50 years were recruited to take Reproknow and demographic questionnaires in two outpatient clinics. Associations between patients' mean total knowledge scores and demographic characteristics were assessed using independent-sample t-tests. Questions were also categorized by topical area, and the percentages were calculated.

Results: The completion rate of questions in Reproknow was 100\% across all users. Median Reproknow scores were significantly higher among rheumatology fellows than among nurses $(p=0.045)$. The 153 patients recruited to the study had at least one of 15 rheumatic diseases. Patients' mean knowledge score was 5.05 (SD 2.24) out of a possible high score of 10 . Patients who were younger, White, and more educated had significantly higher scores than did other patients ( $p$ 's $<0.05$ ). Patients who bore children after their disease diagnosis had higher knowledge scores than did women whose children were born prior to their diagnosis; in contrast, women with histories of surgical sterilization or hysterectomy had lower knowledge scores than other women. Knowledge scores of women who used potentially fetotoxic medications did not vary from the remainder of the sample. Patients demonstrated gaps in knowledge about birth outcomes, contraceptive efficacy, and breastfeeding safety.
\end{abstract}

Conclusions: Initial testing of ReproKnow suggests that it may be a promising tool to assess the reproductive health knowledge of women with diverse rheumatic diseases. Specific knowledge deficits elicited from ReproKnow may be important targets for future educational interventions.

Keywords: Women's health, Reproduction, Contraception, Autoimmune, Pregnancy

\section{Background}

Women with rheumatic diseases may face considerable reproductive health challenges during their childbearing years. Rheumatic diseases such as systemic lupus erythematosus (SLE), Sjogren's syndrome, inflammatory arthritis, the inflammatory myopathies,

\footnotetext{
* Correspondence: birrums@upmc.edu

${ }^{1}$ Division of Rheumatology and Clinical Immunology, Department of Medicine, University of Pittsburgh, 3500 Terrace Street, Pittsburgh, PA 15261, USA

Full list of author information is available at the end of the article
}

and vasculitides have been associated with higher rates of pregnancy-associated mortality and morbidity compared to rates among healthy women [1-5]. Several common anti-rheumatic drugs have well-established teratogenic potential (e.g., methotrexate, mycophenolate mofetil) $[6,7]$ and estrogen-containing contraceptive methods may increase the risk of thromboembolism among women with anti-phospholipid antibodies $[8,9]$.

It is important that women are empowered to make well-informed reproductive decisions that optimize their chances for favorable health outcomes. Such decisions

(c) The Author(s). 2019 Open Access This article is distributed under the terms of the Creative Commons Attribution 4.0 International License (http://creativecommons.org/licenses/by/4.0/), which permits unrestricted use, distribution, and 
may include planning their pregnancies for a time when their diseases are quiescent or adequately controlled on safe anti-rheumatic drugs, or by selecting non-hormonal birth control methods depending on their thrombotic risk - practices that have been shown to improve health outcomes, and that are supported by the American College of Rheumatology (ACR) and the European League Against Rheumatism [10, 11]. In contrast, women who lack knowledge about the relationships between their diseases and pregnancy outcomes may make lessinformed health decisions that have deleterious clinical consequences. For example, a woman who erroneously believes that hormonal contraception is incompatible with her rheumatic disease may avoid using birth control, which could increase her risk for an unintended pregnancy that occurs while her disease is active or while using a fetotoxic anti-rheumatic drug.

Limited available research seems to suggest that some women with rheumatic diseases may lack adequate knowledge to make well-informed family planning decisions, which might eventually culminate in suboptimal reproductive health outcomes. Young women with rheumatoid arthritis (RA) in one qualitative study reported that they struggled to find reliable or relevant information about RA and pregnancy [12]. Young women with inflammatory arthritis in another study relied on unverified blogs, social media, and online forums to find relevant information about arthritis and pregnancy [13].

To develop tailored interventions that ameliorate important gaps in patients' reproductive knowledge, those gaps in knowledge must first be identified. A number of self-administered questionnaires to assess patients' knowledge have been developed across medical disciplines for conditions such as diabetes, inflammatory bowel disease (IBD), asthma, and osteoarthritis [14-17]. These questionnaires are generally paper-based, low-cost, and easy to administer in clinical or research settings. Self-administered questionnaires may minimize physician burden and increase efficiency by identifying patients' specific knowledge gaps prior to the physician encounter; the physician may then educate patients on the knowledge gaps that are most pertinent to their medical conditions. Patients' performance on self-administered assessments have been linked to health behaviors; for example, among young women who did not desire pregnancy, women with lower knowledge scores on a contraception self-administered questionnaire were found to have lower rates of contraception continuation at 6 months' follow-up [18]. This suggests that such self-administered assessments have the potential to identify key gaps in patients' knowledge that predict suboptimal health behaviors, and may be intervened upon to enhance their health outcomes.

Self-administered questionnaires may be particularly valuable for assessing reproductive health knowledge of women with rheumatic diseases in the clinical setting. Several survey-based studies suggest that women with rheumatic diseases infrequently receive reproductive health education or family planning counseling from their providers, even when their disease severity or use of teratogenic anti-rheumatic drugs increases their risk for pregnancy complications [19-22]. Providers who do attempt to clarify what patients know or do not know about their diseases and reproductive health may obtain a highly subjective assessment depending on the questions that they ask or do not ask, or their assumptions about the information that they believe is most relevant to the patient. An objective, standardized questionnaire that assesses multiple dimensions of women's reproductive knowledge may increase the chances that a conversation occurs between providers and patients about key reproductive health issues that might be particularly relevant to the patient.

Few self-administered assessments exist for to assess reproductive health knowledge among women with rheumatic diseases. The Pregnancy in Rheumatoid Arthritis Questionnaire (PIRAQ) is a 17-item questionnaire used in research [23]. The Crohn's and Colitis Pregnancy Knowledge Score (CCP-Know) is a 17-item questionnaire developed for clinical and research settings [24]. CCP-Know scores, in which higher scores indicated better knowledge, were found to be low in a sample of reproductive-age women with IBD, particularly among women who had not experienced a pregnancy after their disease diagnosis - and therefore might not be expected to have the experiential knowledge to answer the CCPKnow questions accurately.

The objectives of the current study were to: 1 ) develop a self-administered questionnaire (ReproKnow) to assess reproductive-age women's knowledge of pregnancyrelated issues in the rheumatic diseases; 2) evaluate the use of ReproKnow in a community-based cohort of women of reproductive age with rheumatic diseases, and describe patients' reproductive knowledge.

\section{Methods}

This study was approved by the University of Pittsburgh Institutional Review Board (PRO17080373).

\section{ReproKnow development}

The content of ReproKnow reflected topics addressed in a patient educational pamphlet produced by the ACR about women's reproductive health that is freely available on its website, and a review article about family planning for women with rheumatic diseases written by several of the current manuscript's authors $[25,26]$. Preliminary questions that addressed heritability of rheumatic diseases, birth outcomes, likelihood of fertility, contraception safety and efficacy, preconception care, 
pregnancy management, lactation/breastfeeding safety, and medication risk/safety, were developed by one of the principal investigators (M.B.T.). Content validation, as defined by Haynes et al., should include population and expert sampling for the initial generation of items and other elements of the scale [27]. To optimize the content validity of ReproKnow, a group of local and national rheumatologists, obstetrician-gynecologists, internists with formal women's health sub-specialization, nurses, a pharmacist, and a survey methodologist, reviewed the questions. Based on their input, six questions were extracted, and the remaining questions were refined. Three female reproductive-age patients with SLE were recruited from an outpatient rheumatology clinic to participate in cognitive, "think-aloud" interviews while they used the preliminary tool. Their feedback about the clarity and content of the tool were used to make additional revisions to the questions and response options.

The current version of ReproKnow includes ten multiple-choice questions that assessed reproductive knowledge across a range of topical domains (Additional file 1). Each question has three to six answer choices, including a "Not Sure" option for all questions. Total, or overall, knowledge scores may range from 0 to 10 , with 10 indicating a perfect score on the assessment. ReproKnow was scored at a third-grade reading level, according to the Flesch-Kincaid reading ease scale [28].

\section{Preliminary validation}

Construct validity assesses the extent to which a scale reflects the abilities of different raters or users. We assessed one dimension of construct validity using a known-groups analysis to compare median total ReproKnow scores of rheumatology fellows to those of rheumatology nurses. We hypothesized that fellows would have higher scores as they have more formal medical training. Rheumatology fellows in their first or second years of training, and rheumatology nurses from two outpatient clinical practices, were invited to complete ReproKnow. Participation was voluntary and the tool was self-administered. Responses were returned anonymously; thus, individual subject characteristics were not collected.

Cronbach's alpha was used to evaluate the internal consistency of ReproKnow; a coefficient of 0.7 or higher is generally considered to be acceptable for established scales, although coefficients of at least 0.6 may be considered for newly-created or preliminary scales [29-31]. We also completed a principal components analysis (PCA) for nominal level variables in order to assess the dimensionality of the scale, with a confirmatory factor analysis for dichotomous variables using the Hull method to determine the number of factors in the scale [32].

\section{Patient sample and data collection}

We administered ReproKnow to female patients aged 18 to 50 years old who were established patients of either a community-based or an academic rheumatology practice affiliated with a large health care system in western Pennsylvania, U.S.A. Patients who had scheduled appointments during the study timeframe were prescreened for eligibility based on age and gender. Eligible patients were approached by study coordinators immediately after their physician visits. Patients who agreed to participate and provided verbal consent were subsequently asked to complete a paper version of the ReproKnow questionnaire.

Women also completed a brief demographic survey, which included questions about age, race/ethnicity, education, disease diagnosis, and current use of biologic or non-biologic anti-rheumatic drugs. We were interested in assessing the reproductive histories of patients in the study to assess whether their knowledge scores might reflect prior experiences with childbearing. Therefore, we inquired about their numbers of biological children, the temporal associations between their childbearing and rheumatic diagnosis, whether their rheumatic diagnosis influenced their childbearing decisions, and if they had a history of surgical sterilization or hysterectomy.

Patients' rheumatic diagnoses were elicited via free text responses, while the remainder of the demographic answer choices were presented in multiple-choice format. Patients were asked to select the anti-rheumatic drugs that they used from a list of commonly-used biologic and non-biologic disease-modifying anti-rheumatic drugs; we categorized medications as potentially teratogenic versus not teratogenic based on published international consensus guidelines $[6,7,33]$.

\section{Statistical analyses}

For construct validation testing, descriptive statistics were used to evaluate the knowledge scores within the rheumatology fellow and nurse groupings. The KruskallWallis test for nonparametric data was used to compare scores between the fellows and nurses.

Among patients who completed ReproKnow, patients' demographic answer choices were collapsed into twocategory variables due to low cell counts for certain responses (i.e., age, race/ethnicity, educational attainment, type of sterilization procedure). Descriptive statistics were used to calculate patients' total knowledge scores. Independent sample t-tests were used to evaluate relationships between patients' demographic characteristics and their mean total knowledge scores. Patients' correct responses to individual ReproKnow questions were also calculated. We also assessed the aggregate percentage of correct scores to the five medication risk questions 
among women who used potentially fetotoxic drugs versus other women in the sample.

Statistical analyses were performed using SPSS version 24 , with two-tailed $p<0.05$ signifying statistical significance. As one of our principal objectives was to generate hypotheses about the associations among knowledge, demographics, and reproductive profiles, we did not adjust for multiple comparisons.

\section{Results}

\section{Subject characteristics}

Construct validation testing was performed with rheumatology fellows and nurses; all six fellows (100\%) and five of the six nurses $(83.3 \%)$ who were invited to complete ReproKnow, chose to participate in the study.

To describe reproductive knowledge among patients, we also recruited and tested ReproKnow among 153 female patients. Patient subjects had a mean age of 38.3 years old (S.D. 8.2), most participants were white (77.0\%), and half of the women had at least a college degree (50.0\%). SLE (23.0\%), Sjogren's syndrome (15.0\%), and RA (14.0\%) were the most common diagnoses, but the sample also included women with undifferentiated connective tissue disease, psoriatic arthritis, spondyloarthritis, antiphospholipid antibody syndrome, inflammatory bowel disease, systemic sclerosis, inflammatory myositis, mixed connective tissue disease, ANCA vasculitis, Takayasu arteritis, Bechet's, and polyarteritis nodosa. Some women did not report their rheumatic diagnosis $(n=12)$ or only reported a nonrheumatic disease diagnosis $(n=22$; e.g., fibromyalgia, unspecified muscle or joint pain, or positive blood test, such as an anti-nuclear antibody). We conducted a sensitivity analysis to evaluate if results differed when we excluded these 34 women from the sample. The results did not change, and as some of the women may have had a rheumatic disease that they chose not to report, or a disease that had not yet been formally diagnosed, we elected to retain all participants in the final sample.

We also assessed the reproductive histories of women in our sample. Three patients were pregnant at the time that they completed ReproKnow. A majority of women had at least one child (64.1\%), and most women experienced all of their pregnancies prior to diagnosis of their rheumatic diseases (75.8\%). Approximately 27\% of women reported that their disease had affected their decision to have any or additional children.

Over one-quarter (25.5\%) of women reported current use of at least one anti-rheumatic drug with fetotoxic potential, which in this sample, included methotrexate, leflunomide, and mycophenolate mofetil [6].

\section{Construct validity}

The completion percentage for all of the questions in ReproKnow was $100 \%$ for rheumatology fellows and nurses. The median total knowledge score for fellows was 8.5 (mean 8.33, S.D. 1.21, range 7-10), and was 7.0 for nurses (mean 6.8, S.D. 0.45, range 6-7). As scores were not normally distributed, nonparametric testing was used to compare median scores between these groups; fellows' scores were significantly higher than were nurses' scores $(p=0.045)$.

\section{Internal consistency}

The Cronbach's alpha statistic for ReproKnow was based on patient responses, and was estimated at 0.62 , which demonstrates moderate internal consistency. To assess if the moderate internal consistency was secondary to multidimensionality in the scale, we subsequently conducted a principal components analysis (PCA) for nominal level variables. The PCA identified four items on two dimensions that had no clinically meaningful relationships. We next removed these four items from the analysis, and subsequently removed all possible combinations of the items from the analysis. This sub-analysis did not change the alpha level. Our factor analysis results were similar to the PCA analysis in that a onefactor solution was also recommended using Hull criteria, but that factor explained a minority of the overall variance $(37.6 \%)$ and several items had either moderately low $(<.40$, item $4 ;<.45$ items 7 and 9$)$, or very low $(<.20$, item 3 ; $<.25$, item 5$)$ loadings.

\section{Patients' total reproductive knowledge scores}

The completion percentage for all of the questions in ReproKnow was $100 \%$ for patients. Patients' total reproductive knowledge scores ranged from 0 to 10, with a median score of 5.0 and mean score of 5.05 (SD 2.24). Patient scores were normally distributed (Fig. 1).

Table 1 presents the associations between patients' characteristics and mean knowledge scores. Younger women had significantly higher total knowledge scores than did older women, Whites had higher scores than non-Whites, and highly-educated women had higher scores than did women with less education $\left(p^{\prime} \mathbf{s}<0.05\right)$. Total knowledge scores did not differ between women who used potentially teratogenic medications and those who used safer medications.

Among parous women in the sample, those patients who were either currently pregnant or who had a child born after their rheumatic diagnosis $(n=23$ for combined group) had higher knowledge scores than did women whose children were born prior to their disease diagnosis $(n=72)(p=0.015)$. Women who had prior sterilization or hysterectomy procedures had lower total knowledge scores than women who did not have these procedures $(p=0.008)$. 


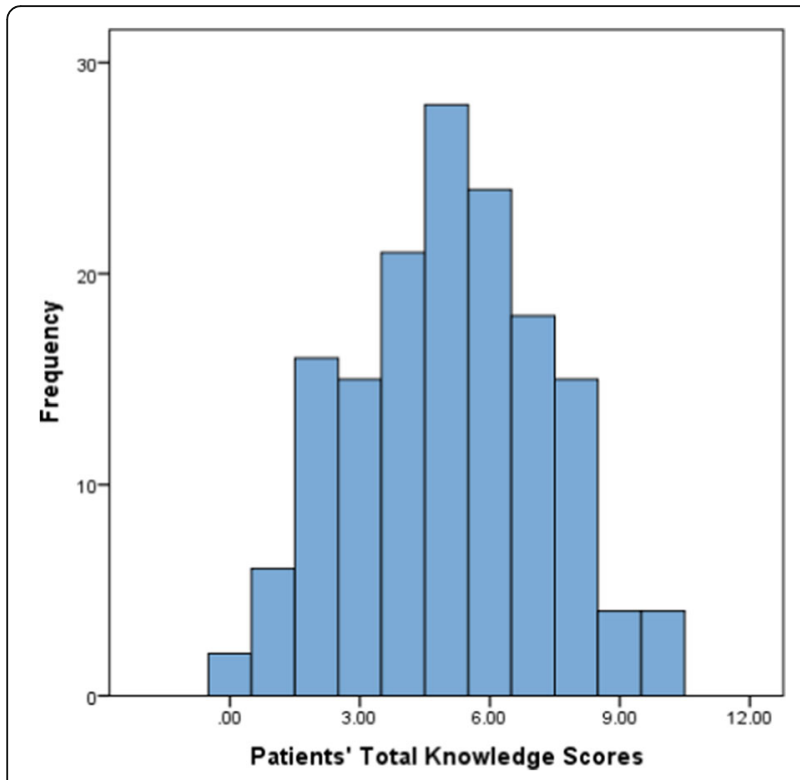

Fig. 1 Distribution of Patients' Total Knowledge Scores. Legend: $N=153$; Mean score: 5.05; Standard deviation: 2.244
Assessment of patients' reproductive knowledge by topic Patients' knowledge scores categorized by topical domains are presented in Table 2. Most patients correctly answered questions about heritability $(75.8 \%)$ and pregnancy management (63.8\%), whereas fewer patients correctly answered questions about birth outcomes $(30.8 \%)$ and breastfeeding safety (28.8\%). Approximately $50 \%$ of respondents correctly answered questions about fertility, contraception, preconception planning, and medication risk.

Among women who used potentially fetotoxic antirheumatic drugs, $54.4 \%$ of questions about medication risk were answered correctly; $55.2 \%$ of these same questions were answered correctly by women who did not use fetotoxic drugs.

\section{Discussion}

The ReproKnow tool was designed to evaluate what women with a broad range of rheumatic diseases know about disease-related reproductive health topics. In our cohort of female, reproductive-age patients, ReproKnow revealed key knowledge gaps related to birth outcomes, safety of lactation, likelihood of fertility, efficacy of contraceptive methods, and medication safety.

The content of ReproKnow was intended to be relevant for reproductive-age women with any rheumatic

Table 1 Patient Demographics and Characteristics and ReproKnow Total Scores

\begin{tabular}{|c|c|c|c|c|}
\hline Characteristics & N (\%) & Total Score Means (S.D.) & Mean Score Difference & $P$ value \\
\hline \multicolumn{5}{|l|}{ Age (years) } \\
\hline $18-34$ & $46(30.1)$ & $5.63(2.22)$ & \multirow[t]{2}{*}{0.83} & \multirow[t]{2}{*}{0.037} \\
\hline $35-50$ & $106(69.3)$ & $4.80(2.23)$ & & \\
\hline \multicolumn{5}{|l|}{ Race } \\
\hline White & $118(77.1)$ & $5.45(2.06)$ & \multirow[t]{2}{*}{1.8} & \multirow[t]{2}{*}{0.002} \\
\hline Non-White & $34(22.2)$ & $3.67(2.37)$ & & \\
\hline \multicolumn{5}{|l|}{ Education } \\
\hline College/Graduate School & $76(49.7)$ & $5.61(5.62)$ & \multirow[t]{2}{*}{1.13} & \multirow[t]{2}{*}{0.002} \\
\hline$<$ High School/Some College & $76(49.7)$ & $4.48(2.21)$ & & \\
\hline \multicolumn{5}{|c|}{ Current Use of Potentially Teratogenic Medication } \\
\hline Yes & $39(25.5)$ & $5.10(2.37)$ & \multirow[t]{2}{*}{0.068} & \multirow[t]{2}{*}{0.42} \\
\hline No & $114(74.5)$ & $5.03(2.21)$ & & \\
\hline \multicolumn{5}{|l|}{ Pregnancy After Diagnosis } \\
\hline Yes & $23(24.2)$ & $6.00(2.28)$ & \multirow[t]{2}{*}{1.16} & \multirow[t]{2}{*}{0.015} \\
\hline No & $72(75.8)$ & $4.83(2.16)$ & & \\
\hline \multicolumn{5}{|c|}{ Disease affected decision to have more children } \\
\hline Yes & $42(28.6)$ & $5.19(2.32)$ & \multirow[t]{2}{*}{0.11} & \multirow[t]{2}{*}{0.19} \\
\hline No & $105(71.4)$ & $5.08(2.07)$ & & \\
\hline \multicolumn{5}{|c|}{ Prior Sterilization or Hysterectomy Procedure } \\
\hline Yes & $40(26.1)$ & $4.25(1.97)$ & -1.09 & \multirow{3}{*}{0.008} \\
\hline & & & & \\
\hline No & 112 (73.2) & $5.34(2.28)$ & & \\
\hline
\end{tabular}

Mean score difference was calculated by subtracting score mean of bolded group from the score mean of un-bolded group 
Table 2 Percentage of Patients' Correct Answers By Question and By Concept Area (Mean Percentage (\%)

\begin{tabular}{|c|c|}
\hline & $\%$ \\
\hline \multicolumn{2}{|l|}{ Heritability } \\
\hline 1. Moms with autoimmune diseases pass their diseases on to their children ( $A^{a}$ : Sometimes) & 75.8 \\
\hline \multicolumn{2}{|l|}{ Birth Outcomes } \\
\hline 1. If I have an autoimmune disease, my baby's chances of being born with a birth defect are (A: Low) & 32.7 \\
\hline 2. If I am pregnant and have a disease flare, my baby may be: (A: Born too early) & 28.8 \\
\hline \multicolumn{2}{|l|}{ Fertility } \\
\hline 1. Most women with autoimmune diseases can get pregnant as easily as other women (A: Yes) & 51.6 \\
\hline \multicolumn{2}{|l|}{ Contraception } \\
\hline 1. Can most women with autoimmune diseases use birth control safely? (A: Yes) & 64.7 \\
\hline 2. Which type of birth control is the best at preventing pregnancy? (A: Intrauterine device (IUD)) & 41.2 \\
\hline \multicolumn{2}{|l|}{ Preconception Planning } \\
\hline $\begin{array}{l}\text { 1. When is the best time for a woman with an autoimmune disease to get pregnant? (A: After her disease is controlled on safe meds } \\
\text { for a few months) }\end{array}$ & 54.2 \\
\hline \multicolumn{2}{|l|}{ Pregnancy Management } \\
\hline $\begin{array}{l}\text { 1. If I find out that I'm pregnant, what should I do next? } \\
\text { (A: Continue my meds until I talk with my doctor) }\end{array}$ & 75.2 \\
\hline 2. If I am pregnant and have a flare of my disease (A: I may need to use meds to protect me and my baby) & 52.3 \\
\hline \multicolumn{2}{|l|}{ Breastfeeding } \\
\hline $\begin{array}{l}\text { 1. Moms with autoimmune diseases who are on safe meds (A: Usually can breastfeed safely, Make breastmilk that is nutritious as other } \\
\text { women's) }\end{array}$ & 28.8 \\
\hline \multicolumn{2}{|l|}{ Medication Risk } \\
\hline 1. Can most women with autoimmune diseases use birth control safely? (A: Yes) & 64.7 \\
\hline $\begin{array}{l}\text { 2. When is the best time for a woman with an autoimmune disease to get pregnant? (A: After her disease is controlled on safe meds } \\
\text { for a few months) }\end{array}$ & 54.2 \\
\hline 3. If I find out that I'm pregnant, what should I do next?(A: Continue my meds until I talk with my doctor) & 75.2 \\
\hline $\begin{array}{l}\text { 4. If I am pregnant and have a flare of my disease } \\
\text { (A: I may need to use meds to protect me and my baby) }\end{array}$ & 52.3 \\
\hline $\begin{array}{l}\text { 5. Moms with autoimmune diseases who are on safe meds (A: Usually can breastfeed safely, Make breastmilk that is nutritious as other } \\
\text { women's) }\end{array}$ & 28.8 \\
\hline
\end{tabular}

Multiple topical domains may be covered by a single question, thus questions may appear more than once in table

${ }^{\mathrm{a}} \mathrm{A}=$ Correct Answer

disease that is treated by a rheumatologist. Several reproductive knowledge assessments exist for specific immune-mediated diseases (e.g., Pregnancy in Rheumatoid Arthritis Questionnaire (PIRAQ) [23], and Crohn's and Colitis Pregnancy Knowledge Score (CCP-Know) [24]. However, such tools are not available for the majority of rheumatic diseases, including diseases with high pregnancy-associated mortality and morbidity, such as systemic lupus erythematosus. This underscores the potential utility of a general tool that assesses knowledge about the shared reproductive risks across rheumatic diseases. Reproknow is also brief, readable, inexpensive, and easy to administer, and may be adaptable for a wide range of research or clinical purposes. The $100 \%$ completion rate among all users underscores its feasibility, particularly in clinical settings.
Our study provides evidence that ReproKnow is a promising tool for assessing reproductive knowledge. First, knowledge scores reflected the level of formal rheumatology training and education of users, including fellows, nurses, and patients, which suggests that it might have acceptable construct validity. Secondly, ReproKnow appeared to reflect patients' knowledge based on their reproductive experiences. Women who had children after their disease diagnosis had higher knowledge scores than did other women; these women likely had some disease-related health counseling during their pregnancies, which may have translated into greater reproductive knowledge. Similarly, lower knowledge scores attained by women who had hysterectomies or sterilization procedures may be reflective of less reproductive health counseling given to women who do not have reproductive potential. 
ReproKnow's relatively low internal consistency might be considered a potential weakness. While Cronbach's alpha is ideal for scales that have multiple response options (e.g., Likert), coefficients may be artificially low for scales with fewer responses [29]. Our interpretation of our findings from PCA and factor analysis were that a single factor solution did not sufficiently explain the variance in the model, and a multiple-factor solution lacked clinical or conceptual meaning. It is possible that future research with ReproKnow involving larger samples of women will reveal a meaningful latent structure. However, the internal consistency, PCA, and factor analysis results may also reflect that ReproKnow is meant to test a broad range of topics across reproductive health, including pregnancy, pregnancy prevention, lactation, and heritability.

Certain patient characteristics were associated with better total knowledge scores, including younger age, White race, and higher educational attainment. However, total knowledge scores or scores on the medication risk questions did not differ between users or non-users of potentially fetotoxic medications. Gaps in reproductive health knowledge among women who use fetotoxic medications may have particularly deleterious effects, especially among women who conceive while using these drugs.

Our analysis also assessed women's knowledge about specific reproductive health domains. Most women overestimated their offspring's risk for congenital anomalies and underestimated the safety of breastfeeding. This finding has been previously reported in studies of the general population, in which many women overestimate the absolute risk of congenital fetal anomalies, and women who use medications for any indication are less likely to breastfeed due to concerns about safety [34, 35]. However, among women with rheumatic diseases, the risk of congenital anomalies does not differ significantly from the general population, including among children who have been exposed to pregnancy-compatible antirheumatic drugs before or during pregnancy [36]. Breastfeeding also appears to be safe for women who use lactation-compatible medications [33]. Our results suggest that some patients may benefit from counseling about risk of congenital anomalies and breastfeeding safety, to help them to make informed decisions about childbearing and breastfeeding.

Approximately half of patients incorrectly answered questions about fertility, the efficacy and safety of contraceptive methods, and preconception planning; these knowledge gaps may affect reproductive decisionmaking and behaviors, and translate into suboptimal reproductive health outcomes. Work by Mosher et al. suggests that women who underestimate their childbearing potential may be more likely to engage in unprotected sex, thus increasing their risk of unintended pregnancy
[37]. In our cohort, only $41.2 \%$ of women were able to correctly identify the most effective contraceptive method of the choices provided. Women with low contraception knowledge may also overestimate the efficacy of methods such as condoms as compared to more efficacious methods (e.g. intrauterine devices), which might further increase their risk of unintended pregnancy even if they do use contraception [38]. More work is needed to assess whether patients with better reproductive knowledge more accurately ascertain reproductive risks associated with their diseases and medications, and make more informed family planning decisions. Several consensus guidelines and reviews are available to help providers educate patients with rheumatic diseases about reproductive health and family planning [11, 33, 39-41].

Our study and analytic design had certain limitations. First, while educational attainment and proportion of white participants in our sample were similar to the demographics of the general western Pennsylvania population, the generalizability of our findings to women from other racial/ethnic or socioeconomic backgrounds may be limited [42]. Our findings may also overestimate the reproductive knowledge of women with rheumatic diseases: women with low functional literacy may have declined participation, and white women, whose knowledge scores were generally higher than other women, were overly represented in our cohort. Therefore, knowledge scores in this cohort may be higher than scores in a more diverse group of women with rheumatic diseases. In addition, while ReproKnow asked women to answer questions based on "most women's" experiences, some women may have answered questions based on their own experiences; for example, women who personally experienced contraceptive failure or infertility, might have answered those questions incorrectly based on their own experiences rather than an understanding of population risk. Our perspective is that a "wrong answer" might actually provide an opportunity for a provider to clarify patients' myths or misconceptions.

In addition, more research is needed to further develop the psychometric properties of ReproKnow. Additional testing of the tool in a variety of clinical (e.g., community-based, academic, or hospital settings, and different geographic locations) or research settings will help to further support the validity and reliability of ReproKnow. Our sample was not racially diverse, and the tool should be explored in more diverse populations of women with rheumatic diseases, perhaps with a wider range of rheumatic diseases. Criterion validity could be explored by assessing whether high scores on ReproKnow translate to better reproductive outcomes over time, perhaps in a longitudinal cohort of women with rheumatic diseases. High scores on a self-administered 
contraception knowledge assessment in one study predicted more consistent contraception use over time among young women who did not desire pregnancy [18]; thus, it is conceivable that patients' reproductive knowledge could be linked to behaviors that optimize patient's reproductive outcomes. This should be explored in future testing of ReproKnow.

In conclusion, ReproKnow is a tool that may help to evaluate the reproductive knowledge of women with a range of rheumatic diseases across a variety of topical domains. Women who use potentially fetotoxic medications appear to be a particularly important target for educational interventions. Given the particularly low scores in contraception, breastfeeding, and birth outcomes, women also may benefit from enhanced knowledge about these topics. Providers should consider identifying addressing specific knowledge gaps in order to provide women with rheumatic diseases with patient-centered, comprehensive care. More research is needed to determine what types of educational interventions may help to close the knowledge gaps in this high-risk population of women.

\section{Conclusions}

The purpose of this study was to develop and test a reproductive knowledge assessment for women with rheumatic diseases, a clinically vulnerable group. Our preliminary validation suggests that ReproKnow has the potential to expose important reproductive health knowledge gaps among these patients. The extent to which these gaps in knowledge predispose to adverse reproductive outcomes should be studied in the future. Additional validation testing should also be conducted in different populations of women with rheumatic diseases.

\section{Supplementary information}

Supplementary information accompanies this paper at https://doi.org/10. 1186/s41927-019-0091-6.

\section{Additional file 1. ReproKnow instrument.}

\section{Abbreviations \\ ACR: American College of Rheumatology; APS: Antiphospholipid antibody syndrome; CCP-Know: Crohn's and Colitis Pregnancy Knowledge Score; IBD: Inflammatory bowel disease; PCA: Principal components analysis; PIRAQ: Pregnancy in Rheumatoid Arthritis Questionnaire; RA: Rheumatoid arthritis; SLE: Systemic lupus erythematosus}

\section{Acknowledgements \\ We acknowledge the patient and provider collaborators who helped to develop and refine ReproKnow. We appreciate Dr. Christian Selinger and Dr. Tanya Meade, who graciously shared their instruments (CCP-Know and PIRAQ, respectively) with our team. We thank the study coordinators, Laurie Hope, Lacy Ruffalo, and Sedin Dzanko. We are greatly appreciative of the patient participants for their time and contributions to this study.}

\section{Authors' contributions}

MBT- Study design, data analysis, manuscript preparation. MEBC- Study design, data analysis, manuscript preparation. SJB- Study design, data analysis, manuscript preparation. GS- Data analysis, manuscript preparation. LY- Data analysis. AC- Data collection. SB- Study design, data analysis, manuscript preparation. All authors have read and approved the final manuscript.

\section{Funding}

M.B.T. was supported by grant number K12HS022989 from the Agency for Healthcare Research and Quality. The funding body was not involved in the design of the study, collection, analysis, or interpretation of data, or authorship of the manuscript.

\section{Availability of data and materials}

Most data and materials are included in this published article and its supplementary files. All other data and materials for this study may be obtained by contacting the corresponding author (birrums@upmc.edu).

\section{Ethics approval}

This study was approved by the University of Pittsburgh Institutional Review Board (PRO17080373). There was minimal risk for participation in the study. Patients were consented verbally; this was approved by the ethics committee as: 1) no information was linked to the patient; 2) no samples were taken from patients; 3) patients' medical charts were not entered; and 4) no follow-up occurred after the initial encounter.

\section{Consent for publication}

Not applicable as data presented are summary data and no individuals are identifiable in this study.

\section{Competing interests}

The authors declare that they have no competing interests.

\section{Author details}

'Division of Rheumatology and Clinical Immunology, Department of Medicine, University of Pittsburgh, 3500 Terrace Street, Pittsburgh, PA 15261, USA. ${ }^{2}$ Duke University Medical Center, Durham, NC, USA. ${ }^{3}$ University of North Carolina Eshelman School of Pharmacy, Chapel Hill, NC, USA. ${ }^{4}$ Division of General Internal Medicine, Department of Medicine, University of Pittsburgh, Pittsburgh, PA, USA. ${ }^{5}$ Division of General Internal Medicine, University of Pittsburgh and UPMC, Pittsburgh, PA, USA. ${ }^{6}$ Veteran's Affairs Pittsburgh Center for Health Equity Research and Promotion, Pittsburgh, PA, USA.

Received: 26 September 2018 Accepted: 26 September 2019 Published online: 21 October 2019

\section{References}

1. Clowse ME, Jamison M, Myers E, James AH. A national study of the complications of lupus in pregnancy. Am J Obstet Gynecol. 2008;199(2):127 e121-6.

2. Clowse ME, Richeson RL, Pieper C, Merkel PA. Pregnancy outcomes among patients with vasculitis. Arthritis Care Res. 2013;65(8):1370-4.

3. Vancsa A, Ponyi A, Constantin T, Zeher M, Danko K. Pregnancy outcome in idiopathic inflammatory myopathy. Rheumatol Int. 2007;27(5):435-9.

4. Ostensen M, Fuhrer L, Mathieu R, Seitz M, Villiger PM. A prospective study of pregnant patients with rheumatoid arthritis and ankylosing spondylitis using validated clinical instruments. Ann Rheum Dis. 2004;63(10):1212-7.

5. Chen JS, Roberts CL, Simpson JM, March LM. Pregnancy outcomes in women with rare autoimmune diseases. Arthritis Rheumatol. 2015;67(12): 3314-23.

6. Flint J, Panchal S, Hurrell A, van de Venne M, Gayed M, Schreiber K, Arthanari S, Cunningham J, Flanders L, Moore L, et al. BSR and BHPR guideline on prescribing drugs in pregnancy and breastfeeding-part I: standard and biologic disease modifying anti-rheumatic drugs and corticosteroids. Rheumatology (Oxford). 2016;55(9):1693-7.

7. Flint J, Panchal S, Hurrell A, van de Venne M, Gayed M, Schreiber K, Arthanari S, Cunningham J, Flanders L, Moore L, et al. BSR and BHPR guideline on prescribing drugs in pregnancy and breastfeeding-part II: analgesics and other drugs used in rheumatology practice. Rheumatology (Oxford). 2016;55(9):1698-702.

8. Sanchez-Guerrero J, Uribe AG, Jimenez-Santana L, Mestanza-Peralta M, LaraReyes $\mathrm{P}$, Seuc AH, Cravioto MD. A trial of contraceptive methods in women with systemic lupus erythematosus. N Engl J Med. 2005;353(24):2539-49. 
9. Petri M, Kim MY, Kalunian KC, Grossman J, Hahn BH, Sammaritano LR, Lockshin M, Merrill JT, Belmont HM, Askanase AD, et al. Combined oral contraceptives in women with systemic lupus erythematosus. N Engl J Med. 2005:353(24):2550-8

10. Andreoli L, Bertsias GK, Agmon-Levin N, Brown S, Cervera R, CostedoatChalumeau N, Doria A, Fischer-Betz R, Forger F, Moraes-Fontes MF, et al. EULAR recommendations for women's health and the management of family planning, assisted reproduction, pregnancy and menopause in patients with systemic lupus erythematosus and/or antiphospholipid syndrome. Ann Rheum Dis. 2017;76(3):476-85.

11. Kavanaugh A, Cush JJ, Ahmed MS, Bermas BL, Chakravarty E, Chambers C, Clowse M, Curtis JR, Dao K, Hankins GD, et al. Proceedings from the American College of Rheumatology Reproductive Health Summit: the management of fertility, pregnancy, and lactation in women with autoimmune and systemic inflammatory diseases. Arthritis Care Res (Hoboken). 2015;67(3):313-25.

12. Ackerman $\mathbf{I N}$, Jordan JE, Van Doornum S, Ricardo M, Briggs AM. Understanding the information needs of women with rheumatoid arthritis concerning pregnancy, post-natal care and early parenting: a mixedmethods study. BMC Musculoskelet Disord. 2015;16:194.

13. Haroun T, Eudy AM, Jayasundara M, Nowell WB, Curtis JR, Whitney White C, Crow-Hercher R, Ginsberg SD, Clowse MEB. Tough Choices: Understanding the Medication Decision-Making Process for Women with Inflammatory Arthritis during Pregnancy and Lactation [abstract]. Arthritis Rheumatol. 2017;69(suppl 10). https://acrabstracts.org/abstract/tough-choices-understanding-themedication-decision-making-process-for-women-with-inflammatory-arthritisduring-pregnancy-and-lactation/. Accessed 17 Oct 2019.

14. Dunn SM, Bryson JM, Hoskins PL, Alford JB, Handelsman DJ, Turtle JR. Development of the diabetes knowledge (DKN) scales: forms DKNA, DKNB, and DKNC. Diabetes Care. 1984;7(1):36-41.

15. Eaden JA, Abrams K, Mayberry JF. The Crohn's and colitis knowledge score: a test for measuring patient knowledge in inflammatory bowel disease. Am J Gastroenterol. 1999;94(12):3560-6.

16. Al Ghobain MO, AlNemer M, Khan M. Assessment of knowledge and education relating to asthma during pregnancy among women of childbearing age. Asthma Res Pract. 2018;4:2.

17. Hill J, Bird H. Patient knowledge and misconceptions of osteoarthritis assessed by a validated self-completed knowledge questionnaire (PKQ-OA). Rheumatology (Oxford). 2007;46(5):796-800.

18. Hall KS, Castano PM, Westhoff CL. The influence of oral contraceptive knowledge on oral contraceptive continuation among young women. J Women's Health (Larchmt). 2014;23(7):596-601.

19. Chakravarty E, Clowse ME, Pushparajah DS, Mertens S, Gordon C. Family planning and pregnancy issues for women with systemic inflammatory diseases: patient and physician perspectives. BMJ Open. 2014;4(2):e004081.

20. Schwarz EB, Manzi S. Risk of unintended pregnancy among women with systemic lupus erythematosus. Arthritis Rheum. 2008;59(6):863-6.

21. Yazdany J, Trupin L, Kaiser R, Schmajuk G, Gillis JZ, Chakravarty E, Schwarz EB. Contraceptive counseling and use among women with systemic lupus erythematosus: a gap in health care quality? Arthritis Care Res. 2011;63(3): 358-65.

22. Toomey D, Waldron B. Family planning and inflammatory bowel disease: the patient and the practitioner. Fam Pract. 2013;30(1):64-8.

23. Meade T, Dowswell E, Manolios N, Sharpe L. The motherhood choices decision aid for women with rheumatoid arthritis increases knowledge and reduces decisional conflict: a randomized controlled trial. BMC Musculoskelet Disord. 2015:16:260.

24. Selinger CP, Eaden J, Selby W, Jones DB, Katelaris P, Chapman G, McDonald C, McLaughlin J, Leong RW, Lal S. Patients' knowledge of pregnancy-related issues in inflammatory bowel disease and validation of a novel assessment tool ('CCPKnow'). Aliment Pharmacol Ther. 2012;36(1):57-63.

25. Birru Talabi M, Clowse MEB, Schwarz EB, Callegari LS, Moreland L, Borrero S. Family planning counseling for women with rheumatic diseases. Arthritis Care Res (Hoboken). 2018;70(2):169-74.

26. American College of Rheumatology. Living well with Rheumatic Disease: Pregnancy and Rheumatic Disease. https://www.rheumatology.org/l-Am-A/PatientCaregiver/Diseases-Conditions/Living-Well-with-Rheumatic-Disease/PregnancyRheumatic-Disease\#sthash\%20.\%20JxTY5YE4.dpuf. Accessed 16 Oct 2019.

27. Haynes SN, Richard DCS, Kubany ES. Content validity in psychological assessment: a functional approach to concepts and methods. Psychol Assess. 1995;7(3):238-47.
28. Kincaid JP, Fishburne RP, Rogers RL, Chissom BS. Derivation Of New Readability Formulas (Automated Readability Index, Fog Count And Flesch Reading Ease Formula) For Navy Enlisted Personnel, vol. 56. Millington: Institute for Simulation and Training; 1975.

29. Peterson RA. A meta-analysis of Cronbach's coefficient alpha. J Consum Res. 1994;21(2):381.

30. Nunnally JC. Psychometric theory. McGraw-Hill: University of Michigan; 1967

31. Moss S, Prosser H, Costello H, Simpson N, Patel P, Rowe S, Turner S, Hatton C. Reliability and validity of the PAS-ADD checklist for detecting psychiatric disorders in adults with intellectual disability. J Intellect Disabil Res. 1998; 42(Pt 2):173-83.

32. Lorenzo-Seva U, Timmerman ME, Kiers HA. The Hull method for selecting the number of common factors. Multivar Behav Res. 2011;46(2):340-64.

33. Gotestam Skorpen C, Hoeltzenbein M, Tincani A, Fischer-Betz R, Elefant E, Chambers C, da Silva J, Nelson-Piercy C, Cetin I, Costedoat-Chalumeau N, et al. The EULAR points to consider for use of antirheumatic drugs before pregnancy, and during pregnancy and lactation. Ann Rheum Dis. 2016;75(5): 795-810.

34. Petersen I, McCrea RL, Lupattelli A, Nordeng H. Women's perception of risks of adverse fetal pregnancy outcomes: a large-scale multinational survey. BMJ Open. 2015;5(6):e007390.

35. Ogbuanu CA, Probst J, Laditka SB, Liu J, Baek J, Glover S. Reasons why women do not initiate breastfeeding: a southeastern state study. Womens Health Issues. 2009;19(4):268-78.

36. Viktil KK, Engeland A, Furu K. Outcomes after anti-rheumatic drug use before and during pregnancy: a cohort study among 150,000 pregnant women and expectant fathers. Scand J Rheumatol. 2012;41(3):196-201.

37. Mosher W, Jones J, Abma J. Nonuse of contraception among women at risk of unintended pregnancy in the United States. Contraception. 2015;92(2): 170-6.

38. Biggs MA, Foster DG. Misunderstanding the risk of conception from unprotected and protected sex. Womens Health Issues. 2013;23(1):e47-53.

39. Birru Talabi M, Clowse MEB, Schwarz EB, Callegari LS, Moreland L, Borrero S. Family planning counseling for women with rheumatic diseases. Arthritis Care Res (Hoboken). 2018;70(2):169-74.

40. Clowse ME. Managing contraception and pregnancy in the rheumatologic diseases. Best Pract Res Clin Rheumatol. 2010;24(3):373-85.

41. Ostensen M. Contraception and pregnancy counselling in rheumatoid arthritis. Curr Opin Rheumatol. 2014;26(3):302-7.

42. United States Census Bureau: American fact finder. 2018. https://factfinder. census.gov/faces/nav/jsf/pages/index.xhtml

\section{Publisher's Note}

Springer Nature remains neutral with regard to jurisdictional claims in published maps and institutional affiliations.

Ready to submit your research? Choose BMC and benefit from:

- fast, convenient online submission

- thorough peer review by experienced researchers in your field

- rapid publication on acceptance

- support for research data, including large and complex data types

- gold Open Access which fosters wider collaboration and increased citations

- maximum visibility for your research: over $100 \mathrm{M}$ website views per year

At BMC, research is always in progress.

Learn more biomedcentral.com/submissions 